\title{
Antibiotic resistance profiles in Panama:Trends from 2007 to 2013
}

\begin{abstract}
Background: Bacterial resistance has become an important public health problem worldwide. In hospital settings, the effects are devastating being a common cause of death. In Panama, bacterial resistance is a matter national sanitary concern. The objective is to describe Panamanian antibiotic resistance profiles trends (2007-2013) and to define the existence and scope of nosocomial infection committees in public hospitals.
\end{abstract}

Methods: A review of the Central Reference Public Health Laboratory from the Gorgas Memorial Institute for Health Studies national antibiotic resistance database to 2007-2013 was performed. A descriptive, cross-sectional study on healthcare associated infections or nosocomial infections committees and/or pharmacovigilance or pharmacotherapy committees was accomplished on 34 hospitals from the public Panamanian sector.

Results: Gram-negative bacilli (E. coli, K. pneumoniae, Acinetobacter spp., and $P$. aeruginosa) had elevated yet sustained resistance patterns against multiple antimicrobials in Panama. Up to $28 \%$ of $E$. coli was resistance to cephalosporin while resistance to quinolones and sulfonamides was as far as $66 \%$ and $71 \%$, respectively. $K$. pneumoniae demonstrated a stable yet elevated rate of resistance against the majority of antimicrobials with exception of aminoglycosides and carbapenems. Acinetobacter spp. were the most resistant bacteria in Panama demonstrating stable elevated resistance trends for all the studied antimicrobials, being the only available therapeutic options $\beta$-lactamase inhibitors and aminoglycosides with a resistance up to $66 \%$ and $40 \%$, respectively. $P$. aeruginosa was more susceptible to penicillins with up to $31 \%$ of resistance, and third generation cephalosporins with as far as $47 \%$ of resistance. For carbapenems, resistance was maintained between $21 \%$ and $42 \%$. S. aureus susceptibility to vancomycin was maintained at $100 \%$. Only $68 \%$ of public Panamanian hospitals have active nosocomial infection committees and from those $74 \%$ have Pharmacotherapeutics and pharmacovigilance commissions. None of the committees had epidemiologist or microbiologist among their participants.

Conclusions: Pseudomonas aeruginosa and Acinetobacter spp are the most resistance bacteria in Panama. It is necessary to strengthen the compliance and operations measures of Nosocomial and Pharmacotherapeutics committees and to emphasize in a greater participation of critical specialists. More education on the rational use of antibiotics should be promoted to control bacterial resistance.

Keywords: bacterial drug resistance, Panamá, antibiotic resistance, antibacterial agents, nosocomial infection, drug therapy, infection control
Volume 6 Issue 5 - 2017

\author{
José Moreno,' Eric Conte,' Maribel \\ Tribaldos, ${ }^{\text {Y }}$ ' \\ Zamorano, ${ }^{3}$ Beatriz Gómez,' Julio Toro ${ }^{3}$ \\ 'Gorgas Memorial Institute for Health Studies, Panama \\ ${ }^{2}$ Pharmacy School, University Latina of Panama, Panama \\ ${ }^{3}$ Social Security, Panama
}

Correspondence: Eric Conte, Gorgas Memorial Institute for Health Studies, Panama, Email econte@gorgas.gob.pa

Received: August 07, 2018 | Published: September 07, 2018

\section{Introduction}

Infectious diseases stand out for their ability to have profound impact on the human species. Since the introduction of penicillin in 1943, antibiotics have revolutionized their treatment. However, antimicrobials have an inherent weakness, the organisms against which they are directed almost invariably evolve mechanisms of resistance. ${ }^{1}$ The emergence or resistance may take place irrespectively of the presence of antibacterial agents. Nonetheless, it is the exposure to these medications what provides the necessary selective pressure for the rise and spread of resistant pathogens. Therefore, the force behind the increasing rates of antibiotic resistance is found on the misuse and abuse of antibacterial agents. ${ }^{2}$ Antibiotic resistance is a growing global public health challenge that could undue decades of progress in decreasing morbidity and mortality associated to infectious diseases. ${ }^{3}$ Moreover, the emergence of antibiotic-resistant bacteria at a rate that exceeds drug discovery threatens to end an age of unparalleled achievements in modern medicine. ${ }^{4}$ The 2015 World
Health Organization Global Action Plan on Antimicrobial Resistance posed five strategic objectives:

a) To improve awareness and understanding of antimicrobial agents.

b) To strengthen knowledge through surveillance and research.

c) To reduce the incidence of infection.

d) To optimize the use of antimicrobial agents.

e) To ensure sustainable investment in countering antimicrobial resistance, all aimed at decrease the associated burden. ${ }^{5}$

The Center for Disease Control and Prevention conservatively estimated that at least 23,000 people die annually in the United States as a result of an infection with an antibiotic-resistant organism and that more than 2 million become sickened. ${ }^{6}$ In addition, only in direct costs, the overall crude economic burden of antibiotic resistance has been 
estimated to be between $€ 1.5$ billion (2007) in Europe and $\$ 55$ billion (2000) in United States. ${ }^{7}$ In low-income and middle-income countries, antibiotic use is increasing, with rising rates of hospitalization, and high prevalence of hospital infections. This burden of resistance if probably associated with longer duration of illness and higher rates of mortality in patients with resistant infections, increasing cost of treatment for resistant infections, and inability to do procedures that rely on effective antibiotics to prevent infection. ${ }^{8}$ It has been estimated that between $38.7 \%$ and $50.9 \%$ of pathogens causing surgical site infections and $26.8 \%$ of pathogens producing infections after chemotherapy are resistant to standard prophylactic antibiotics in the United States. Moreover, a 30\% reduction in this efficacy could result in 120,000 additional surgical site infections and infections after chemotherapy per year, and 6300 infection related deaths. ${ }^{9}$

In hospital settings, the concurrence of high antibiotic consumption, critically ill patients and a permanent influx of pathogenic species within the healthcare setting nurtures the development of resistance. ${ }^{2}$ It has been described that in United States, $4 \%$ of hospitalized patients have at least one healthcare associated infection, being pneumonia and surgical site infection the most common ones, followed by gastrointestinal, urinary tract and primary bloodstream infections. Furthermore, the most common pathogens reported are $C$. difficile, $S$. aureus, K pneumoniae and E. coli. ${ }^{10}$ Strategies to successful control bacterial resistance in hospital settings include among others, surveillance of antibiotic use and to detect resistance in human beings and animals; policies for prudent antibiotic use in human being and animals; standardized infection control policies and sufficient staffing; and antibiotic stewardship programs in hospitals and other healthcare facilities. ${ }^{8,11,12}$ Surveillance of bacterial resistance programs generate essential information, which promotes and directs stewardship activities. The later can minimize the unwanted consequences of antimicrobial use by reducing antibiotic use by $20-40 \%$, incidence of health care associated infections, length of hospital stay, and prevalence of bacterial resistance. ${ }^{2,4}$

The Panamanian Ministry of Health resolution number 1392 approved the national stewardship program for epidemiologic surveillance of nosocomial infections and there is a national guideline for antibiotics prescription in Panamá. The main strategy presented is to strength the national epidemiologic surveillance of nosocomial infections through the creation or reactivation of nosocomial committees in each hospital and by building a laboratory network for research of bacterial resistance patterns..$^{13}$ The surveillance of bacterial resistance patterns is performed by the Central Reference Public Health Laboratory (LCRSP) of the Gorgas Memorial Institute for Health Studies (ICGES). This laboratory maintains the epidemiologic surveillance network in clinic microbiologic (RNVEMC) since 2000 , in subscription to the Latin-American network of antimicrobial resistance (ReLAVRA) and report susceptibility patterns to nosocomial committees all over the country. In addition, the LCRSP receives between 10 and $20 \%$ of the antibiotic resistant samples from all over the country for quality control.

In the ReLAVRA 2010 annual report, the bacteria that resulted with the highest rates of resistance in hospital setting were $E$. coli, $K$. Pneumoniae, Enterobacter spp, Staphylococcus aureus, Acinetobacter baumanni, and Pseudomonas aeruginosa.${ }^{14}$ Moreover, the 2013 report from the Emerging Markets Resistance Surveillance Programme LATAM EMRS nations demonstrated variable, yet elevated levels of resistance especially among Enterobacteriaceae ( $\beta$-lactamasemediated), P. aeruginosa and Acinetobacter spp. Methicillin resistant Staphylococcus aureus (48\%), vancomycin-resistant Enterococci
$(15 \%)$ and multidrug resistant S. pneumoniae were also regional therapeutic challenges needing immediate epidemiologic attention. ${ }^{15}$ The objective of this study is to describe Panamanian antibiotic resistance profiles trends from 2007 to 2013 and to describe the existence and scope of nosocomial infection committees in public Panamanian hospitals.

\section{Methods}

A review of the Central Reference Public Health Laboratory (LCRSP) from the Gorgas Memorial Institute for Health Studies (ICGES) national antibiotic resistance database from 2007 until 2013 was performed. This database includes information derived from 32 laboratories from the country (public and private health installations) located all over the Panamanian territory. Participating laboratories receive suspicious samples from hospitalized patients. These samples are studied for bacterial presence. When cultures are positive, bacterial identification and antibiotic sensibility are analyzed. Vitek 2 compact $^{\circ}$ (bioMérieux ${ }^{\odot}$ ) is utilized for both test, with identification cards GN and sensibility cards AST 249, 250 or similar. Digitalized yearly information is received by the LCRSP. Data analysis is performed with $\mathrm{WHONET}^{\circ}$ software.

For the purposes of this analysis, six bacterial organisms were studied: Escherichia coli, Klebsiella pneumoniae, Enterobacter, Staphylococcus aureus, Acinetobacter spp. (A. baumanni, A. coalcaceticus, A. genomospecies), Pseudomonas aeruginosa. An isolate was considered resistant to an antimicrobial agent when tested and interpreted as resistant in accordance with the clinical breakpoint criteria used by the local laboratory. Data was expressed as a resistance percentage. In addition, $95 \% \mathrm{CI}$ were calculated. The statistical significance of temporal trends of microbiologic resistance percentages was calculated based on the presented data using the Cochran Armitage test. P-values $<0.05$ were considered statistically significant. In addition, a descriptive, cross sectional study was performed. From the 34 hospitals belonging to the public Panamanian sector, clinics with known healthcare associated infections or nosocomial infections committees and/or pharmacovigilance or pharmacotherapy committee were included. Two active members of each committee answered a structured questionnaire on one programmed visit. Analysis was performed using Microsoft Access and Excel 2010.

\section{Ethical aspects}

The study protocol was approved by the ICGES Bioethics Committee and by the Ministry of Health and Social Security Educational and Research Departments. All the participant centers gave administrative authorizations for the study.

\section{Results}

\section{Antibiotic resistance trends}

All trends for antibiotic resistance profiles for clinically important bacteria in Panama are presented in Table 1.

\section{Escherichia coli}

During the studied period, the LCRSP received around 3000 reports of resistance per year, being the bacteria with the higher number of isolates studied. Between 2007 and 2013 there were no statistically significant variations in the susceptibility trends of the Escherichia coli isolates. Up to $28 \%$ of Escherichia coli strains were resistance to third generation cephalosporins (ceftazidime, cefotaxime) and up 
to $24 \%$ to fourth generation cephalosporin (cefepime). Similarly, resistance to quinolones (nalidixic acid, ciprofloxacin) and sulfonamides (trimethoprim-sulfamethoxazole) was as far as $66 \%$ and $71 \%$, respectively. Carbapenems and nitrofurans resistance remained rare in the analyzed sample.

\section{Klebsiella pneumoniae}

From 2007 to 2013, the LCRSP received up to 1865 resistance reports for Klebsiella pneumoniae per year. This bacteria demonstrated a stable yet elevated rate of resistance against the majority of antimicrobials with exception of aminoglycosides (amikacin) and carbapenems (imipenem and meropenem), which susceptibility rates kept elevated. Nevertheless, although meropenem susceptibility was high $(94.2 \%)$, there was statistical significant tendency for its increment during the studied period.

\section{Enterobacter species}

Differing from the other observed microbial, for Enterobacter spp isolates the studied period was 2008-2013, because the data was so few that it was not significant. The LCRSP received around 900 isolates per year. During the studied period, the strains showed statistically stable patterns of resistance to all the studied antimicrobials. Antibiotics that showed higher rates of resistance were $\beta$-lactamase inhibitors (piperacillin-tazobactam), up to $22 \%$; third generation cephalosporins (cefotaxime and ceftazidime), as far as $26 \%$; sulfonamides (trimethoprim-sulfamethoxazole) up to $30 \%$, and nitrofurans (nitrofurantoin) up to $23 \%$. Carbapenems sensibility was over $95 \%$ during the studied period.

\section{Acinetobacter species}

Up to 3020 isolates were studied yearly. Acinetobacter species were the most resistant bacteria in Panama. A. baumanni, A. coalcaceticus and $A$. genomespecies, demonstrated stable elevated resistance trends for all the studied antimicrobials. From the studied antibiotics, the still available therapeutic options were $\beta$-lactamase inhibitors (ampicillinsulbactam) with a resistance up to $66 \%$ and aminoglycosides (amikacin) with a resistance of up to $40 \%$. Carbapenems resistance was up to $75 \%$.

\section{Pseudomonas aeruginosa}

Between 2007 to 2013, the LCRSP received around 2000 isolates per year. Resistance trends for Pseudomonas aeruginosa were stable yet elevated for the studied period for all the studied antibiotics. Pseudomonas aeruginosa strain was more susceptible to penicillins (piperacillin) with up to $31 \%$ of resistance, and third generation cephalosporins (ceftazidime) with as far as $47 \%$ of resistance. For carbapenems, (meropenem) resistance was maintained between $21 \%$ and $42 \%$ while for $\beta$-lactamase inhibitors (piperacillin-tazobactam) resistance was between $4 \%$ and $27 \%$.

\section{Staphylococcus aureus}

The number of isolates studied per year varied between 1937 and 2784. Concerning 2007 and 2013 up to $99 \%$ of Staphylococcus aureus isolates were resistant to penicillin being this trend statistically significant. In addition, as far as $55 \%$ and $35 \%$ of strains were resistant to oxacillin and second-generation cephalosporin (cefoxitin) considered methicillin resistant (MRSA). Lastly, it was demonstrated a sustained resistance pattern for macrolids (erythromycin), up to $30 \%$; lincosamides (clindamycin), as far as $28 \%$ and quinolones (ciprofloxacin) up to $23 \%$. Susceptibility to vancomycin was maintained at $100 \%$ during the studied period.

\section{Nosocomial infection committees}

From the 34 hospitals belonging to the Panamanian public healthcare, 23 hospitals $(68 \%)$ have active nosocomial infections committees. Member disciplines include medical doctors and nurses $(100 \%)$, pharmaceutics $(57 \%)$, medical technologists $(83 \%)$ and administrative staff or cleaning staff $(70 \%)$, while the frequency of meetings were mostly in monthly $43 \%$ ) or weekly $(60 \%)$ bases (Table 2 ). With regards to operative actions resulting from the functioning of committees, $100 \%$ referred that records of each meetings were kept. In addition, resistance surveillance manuals were prepared as well as data collection sheets. Among committees functions prevalence or incidence studies, outbreak identification, preventive and isolation measures and surveillance of hospital and ambulatory wards were mentioned. Lastly, 96\% of committees referred having history of bacterial resistance in their institutions. From the 23 hospitals that had nosocomial infections committees, 17 (74\%) also had pharmacovigilance or pharmacotherapy commissions. These boards included medical doctors $(94 \%)$, nurses $(71 \%)$, pharmaceutics $(88 \%)$, medical technologists $(41 \%)$ and administrative staff $(76 \%)$. Reunions were carried out mostly on monthly bases. All of the pharmacovigilance of pharmacotherapy commissions produced guidelines for antibiotic prescription control while only $30 \%$ referred having therapeutic protocol guidelines. In addition, only $30 \%$ had enforced therapeutic protocol guideline use whilst 53\% routinely reported antibiotic consumption in their hospitals.

Table 2 Existence and scope of nosocomial infection committees in public Panamanian hospitals

\begin{tabular}{llc} 
Nosocomial infections committees & & \\
\hline Members discipline & n & $\%$ \\
\hline Doctor & 23 & 100 \\
Nurse & 23 & 100 \\
Pharmacist & 19 & 57 \\
Medical technologist & 16 & 83 \\
Administrative & 16 & 70 \\
Cleaning staff & 13 & 70 \\
Others: biosafety, nutritionist, nurse technician, laundry & 13 & 57 \\
\hline Meeting frequencies & & \\
\hline Weekly & 7 & 30 \\
Biweekly & 3 & 13 \\
Monthly & 10 & 43 \\
Bimonthly & 23 & 100 \\
\hline Quarterly & 4 & 9 \\
\hline Operative actions resulting from its functioning & & \\
\hline & & \\
\hline
\end{tabular}


Table continued...

\begin{tabular}{lcc} 
Nosocomial infections committees & & \\
\hline Members discipline & n & $\%$ \\
\hline Resistance surveillance manuals & 20 & 87 \\
Data collection sheets & 22 & 96 \\
Prevalence or incidence studies & 14 & 61 \\
Outbreak identification & 22 & 96 \\
Preventive and isolation measures & 23 & 100 \\
Surveillance of hospital and ambulatory wards & 23 & 100 \\
History of bacterial resistance in its institution & 22 & 96 \\
Pharmacovigilance or pharmacotherapy commission & 17 & 74 \\
\hline
\end{tabular}

\section{Members discipline}

\begin{tabular}{|c|c|c|}
\hline Doctor & 16 & 94 \\
\hline Nurse & 12 & 71 \\
\hline Pharmacist & 15 & 88 \\
\hline Medical technologist & 7 & 41 \\
\hline Administrative & 13 & 76 \\
\hline Others: health statistics, dentist, radiology technician & 6 & 35 \\
\hline \multicolumn{3}{|l|}{ Meeting frequencies } \\
\hline Weekly & 3 & 18 \\
\hline Biweekly & 2 & 12 \\
\hline Monthly & 8 & 47 \\
\hline Bimonthly & 2 & 12 \\
\hline Quarterly & I & 6 \\
\hline Does not have meetings & I & 6 \\
\hline
\end{tabular}

$\begin{array}{llll}\text { Guidelines for antimicrobial prescription control } & 17 & 100\end{array}$

Therapeutic protocol guidelines

Enforcement of therapeutic protocol guidelines

Routine antibiotic consumption reports

953

\section{Discussion}

Antibiotic resistance is a major global problem that can only be tackled through a comprehensive approach that includes drug discovery and development, sustainable antibiotic use policy and disease prevention strategies. ${ }^{16}$ Now days, at least some clinical isolated of many pathogenic bacteria species such as Mycobacterium tuberculosis, Neisseria gonorrhoeae, Enterococcus faecium, Staphylococcus aureus, Klebsiella pneumonia, Acinetobacter baumanni, Pseudomonas aeruginosa and Enterobacter spp., are now resistant to most antibiotics. ${ }^{17}$ In health care settings, the situation becomes even worst, considering that hospitals are complex environments that can enable the transmission of microorganism and outbreaks. Factors associated with nosocomial infections are patient condition of vulnerability, immunosuppression, and use of medications, facility design, and multitude of life-saving invasive procedures, contamination of hospital environment with organism including resistant bacteria, and close proximity of patients, as well as frequent contact with health care personnel. ${ }^{18}$

The presented manuscript showed that gram-negative bacilli, specifically Escherichia coli, Klebsiella pneumoniae, Acinetobacter spp., and Pseudomonas aeruginosa were the clinical relevant bacteria associated with nosocomial infections, with elevated yet sustained resistance patterns against multiple antimicrobials in Panama. These finding generates foremost concern for healthcare professionals, given that $\beta$-lactamase inhibitors, cephalosporins and quinolones are the antibiotics mostly affected. In line with these data, the occurrence of extremely drug-resistant and even totally drug-resistant phenotypes has been consistently reported among bacteria related to healthcare associated infections such as Pseudomonas aeruginosa, Acinetobacter spp., and Klebsiella pneumoniae. On the other hand, multidrug-resistant microorganisms are increasingly prevalent in the community, including Escherichia coli. ${ }^{19}$ As described in the results, Escherichia coli resistance profile included less than $80 \%$ sensitivity for third and fourth generation cephalosporins, and fewer than $40 \%$ for quinolones and sulfonamides. Equivalent resistance rates for cephalosporins and quinolones have been reported by several European Countries, especially the ones on southern and southeast Europe, ${ }^{20,21}$ and Mexico. ${ }^{22}$ With respect to carbapenems, Panamanian resistance trend remained almost undetectable. These findings coincide with those from Europe, which reported resistances up to $1.2 \%,{ }^{20}$ and Mexico where carbapenems sensibility remains up to 99\%. ${ }^{22}$ However, attention should be placed on carbapenem resistance given that carbapenemase producing Enterobacteriaceae are becoming widespread and given that an increase in combined resistance and the elevated frequency of extended spectrum $\beta$-lactamase producing isolates may lead to a proliferation use of carbapenems, thus favoring dissemination of resistance. ${ }^{20}$

Data presented also showed that Klebsiella pneumoniae isolates had elevated resistance (over 20\%) against the majority of antibiotics except for aminoglycoside and carbapenems. These bacteria has been an increasing concern in Latin America since the majority of infections related with it are healthcare associated and because these microorganism can be spread rapidly between colonized or infected persons or fomites. ${ }^{20} \mathrm{~A}$ report on susceptibility rates in various Latin American nations indicated that Klebsiella spp. presented elevated resistance rates against the majority of medications, with only four drugs inhibiting over $80 \%$ of isolates (tigecicline, colistin, meropenem and amikacin). ${ }^{23}$ Close monitoring of carbapenem resistance should be paid in Panamanian hospitals, since this trend has been statistically increasing over time. During 2011, an outbreak of carbapenemresistant Klebsiella pneumoniae infection in a public hospital in Panama produced over 50 deaths. ${ }^{24}$ As a result, the Ministry of Health prepared a resolution including case definitions and dictated 
mandatory report on this infection. ${ }^{25}$ Similar outbreaks have occurred in different countries. Specifically in the Mediterranean region, were carbapenem-resistant Klebsiella pneumoniae has become endemic. ${ }^{26}$ Consequences of this tendency can be devastating since very few therapeutic options are left for patients infected with multidrugresistant $K$. pneumoniae with additional resistance to carbapenems, and mortality can therefore become elevated. ${ }^{20}$ The resistance pattern of Enterobacter spp presented in this analysis resulted stable, being $\beta$-lactamase inhibitors, cephalosporins, sulfonamides and nitrofurans the antibiotics with lower rates of activity while carbapenems remained with over 95\% sensibility. Similar results have been described in Latin America. ${ }^{23}$ However, in other countries like Taiwan, carbapenem resistant Enterobacter is a worrisome threat that is present not only on hospitals but in the community. ${ }^{27}$

Pseudomonas aeruginosa and Acinetobacter spp were the most resistant bacteria in Panama during the studied period. $P$. aeruginosa had sensibility rates lesser than $70 \%$ and $60 \%$ for penicillin and cephalosporins, respectively while for carbapenem and $\beta$-lactamase inhibitors susceptibility was maintained around $60 \%$ and $70 \%$. In line with these results, combined resistance to multiple antibiotics has also been reported in Europe, Latin America and Mexico. ${ }^{20,22,23}$ Since $P$. aeruginosa carries intrinsic resistance to a number of antimicrobials classes, any additional acquired resistant can severely limit the therapeutic options available for treating a serious infection..$^{20}$ On the other hand, Acinetobacter spp presented susceptibility rates lower than $60 \%$ for aminoglycosides and $44 \%$ for $\beta$-lactamase inhibitors and elevated resistance to carbapenems. Concurring with these data, in Brazil, resistance to carbapenem and $\beta$-lactamase inhibitors has been described to be over $96 \%$ while aminoglycoside sensibility was around $20 \%{ }^{28}$ These data does not surprise given that in the last decade, Acinetobacter spp emerged as a major nosocomial pathogen complex worldwide because of it remarkable ability to develop resistance to broad-spectrum antibiotics. ${ }^{29}$ Moreover, in healthcare environment, bacteria can persist for long periods and is notoriously difficult to eradicate once established. ${ }^{20}$

Lastly, in Panama Staphylococcus aureus resistance trend indicated that MRSA was present in around $55 \%$ of isolates while there was a sustained $100 \%$ susceptibility to vancomycin. Latin America susceptibility rates are similar, $47.8 \%$ of MRSA and complete susceptibility to vancomycin, ${ }^{23}$ while in Europe MRSA presence varies from $0.9 \%$ to $56.6 \%$, with a significant tendency for decreasing. ${ }^{20}$ Nevertheless, the proportion of community onset infections caused by MRSA clones has increased, indicating transfer of healthcare associated MRSA into the community ${ }^{20}$ Considering that misuse and abuse of antibiotics is probably a paramount contributor to this crisis, worldwide organizations and governmental institutions from diverse countries have created strategies to promote the rational use of such drugs, including stewardship programs. ${ }^{30}$ Nevertheless, antibiotics continue to be among the most commonly used therapeutic agents in developed countries like United States, being responsible for $12 \%$ of ambulatory healthcare prescription. As well, antibiotics account for nearly $\$ 100$ billion in annual medication expenditure. ${ }^{31}$ In developing nations, the problem is also present. In Panamá, where medication expenditure is significantly increasing on yearly bases, antibiotics account for $12.43 \%$ of all pharmaceutical expense reaching over 50 million for 2012. ${ }^{32}$

Antimicrobial stewardship has emerged as a global priority, emphasized through international action plans, legislation and national guidelines that promote the judicious use of antimicrobials to preserve their future effectiveness. ${ }^{33}$ Infection prevention programs are now a standard in healthcare showing a $32 \%$ reduction on hospital acquired infections in hospitals with established programs compared with the $18 \%$ increases in infection in hospitals without. ${ }^{34}$ Organizations striving to offer quality care must integrate infection prevention and control programs and antimicrobial stewardship improvement initiatives into a wider, comprehensive safety culture. Highly effective infection prevention surveillance programs should include surveillance of nosocomial infections with feedback to healthcare workers, an intense infection control program including best practices with sterilization, disinfection, asepsis and handling of medical devices, an infection prevention nurse to supervise the program and a physician epidemiologist or microbiologist with special skill in infection prevention..$^{35}$ Albeit regulations are enforced in Panama, based on the collected data, only $68 \%$ of public Panamanian hospitals have active nosocomial infection committees and from those $74 \%$ have Pharmacotherapeutics and pharmacovigilance commissions. Moreover, none of the active committees reported having an epidemiologist among their members or a microbiologist. These figures are worrisome since Panama has resistance trends consistently with developed countries however, its stewardship programs does not comply with international and national regulations. Much work should be placed on guaranteeing the active participation of nosocomial infection committees in every healthcare institution in the Country.

This study has the limitation of assuming that the verification and quality of the susceptibility-testing format has being considered by all the laboratories belonging to the surveillance network. Moreover, since the LCRSP only receives digital yearly reports, it is presumed that neither duplicates nor input errors are present. Nevertheless, to authors' knowledge, this is the first effort to analyze antibiotic resistance trends in Panama using surveillance data from the LCRSP, including multiple healthcare facilities within the country, which resulted in a random distribution of sites that is representative of all the Panamanian territory.

\section{Conclusion}

Antibiotic resistance trends from 2007 until 2013 are similar to those reported worldwide. Pseudomonas aeruginosa and Acinetobacter spp are the most resistance bacteria presenting low susceptibility to the majority of antibiotics. It is necessary to strengthen the compliance and operations measures of Nosocomial and Pharmacotherapeutics committees and to emphasize in a greater participation of critical specialists. More education on the rational use of antibiotics should be promoted to control bacterial resistance.

\section{Acknowledgements}

This research was supported by The Gorgas Memorial Institute for Health Studies. We thank the General Direction for their support for the accomplishment of this study, as well as the technical and administrative personnel that collaborated on the organization and execution of the activities carried out during the investigation. Special thanks to the Microbiology Group of the ICGES: Raquel de Bolaños, Ruben Ramos, Marlenys Fernandez, Gloriela Villarreal and Wendy Castillo.

\section{Conflict of interest}

Author declares that there is no conflict of interest. 


\section{References}

1. Fauci AS, Morens DM. The perpetual challenge of infectious diseases. $N$ Engl J Med. 2012;366(5):454-461.

2. Roca I, Akova M, Baquero F, et al. The global threat of antimicrobial resistance: science for intervention. New Microbes New Infect. 2015;6:22-29.

3. Alsan M, Schoemaker L, Eggleston K, et al. Out-of-pocket health expenditures and antimicrobial resistance in low-income and middle-income countries: an economic analysis. Lancet Infect Dis. 2015;15(10):1203-1210.

4. Perry J, Waglechner N, Wright G. The Prehistory of antibiotic resistance. Cold Spring Harb Prespect Med. 2016;6(6):a025197.

5. Global Action Plan on Antimicrobial Resistance. Geneva: World Health Organization; 2010. 28 p.

6. Arias C, Murray B. A New antibiotic and the Evolution of Resistance. $N$ Engl J Med. 2015;372(12):1168-1170.

7. Gandra S, Barter DM, Laxminarayan R. Economic burden of antibiotic resistance: how much do we really know? Clin Microbiol Infect. 2014;20(10):973-979.

8. Laxminarayan R, Duse A, Wattal C, et al. Antibiotic resistance-The need for global solutions. Lancet Infect Dis. 2013;13(12):1057-1098.

9. Tellinat A, Gandra S, Barter D, et al. Potential burden of antibiotic resistance on surgery and cancer chemotherapy antibiotic prophylaxis in the USA: a literature review and modelling study. Lancet Infect Dis. 2015;15(12):1429-1437.

10. Magill SS, Edwards JR, Stat M, et al. Multistate Point Prevalence of health care associated infections. $N$ Engl J Med. 2014;370(13):1198-1208.

11. Lee JH, Park KS, Karim AM, et al. How to minimize antibiotic resistance. The Lancet. 2016;16(4):17-18.

12. Ponce de Leon Rosales S, Arredondo Hernandez R, Lopez Vidal Y. Resistence to antibiotic: A serious global problem. Gac Med Mex. 2015;151(5):632-639.

13. Gaceta oficial digital. Panama: Gobierno Nacional; 2010. 54 p

14. Informe anual de la Red Latinoamericana de Vigilancia de la Resistencia a los Antibióticos, 2010. Washington, DC: Organización Panamericana de la Salud; 2013. 210 p.

15. Jones RN, Guzman-Blanco M, Gallegos B, et al. Susceptibility Rates in Latin American Nations: Report from the Emerging Markets Resistance Surveillance Programme. ECCMID; 2013.

16. Watkins RR, Bonomo RA. Overview: Global and local impact of antibiotic resistance. Infect Dis Clin N Am. 2016;30(2):313-322.

17. Nathan C, Cars O. Antibiotic resistance-Problems, progress and prospects. New Engl J Med. 2014;371(19):1761-1763.

18. Sood G, Perl TM. Outbreaks in health care settings. Infect Dis Clin N Am. 2016;30(3):661-687.

19. Rossolini GM, Arena F, Pecile P, et al. Update on the antibiotic resistance crisis. Curr Opin Pharmacol. 2014;18:56-60.

20. Antimicrobial resistance surveillance in Europe 2014: Annual report of the European Antimicrobial Resistance Surveillance Network (EARSNet). Stockholm: European Centre for disease prevention and control; 2015. $130 \mathrm{p}$
21. Abat C, Chaudet H, Colson P, et al. Real Time Microbiology laboratory surveillance system to detect abnormal events and emerging infections, Marseille, France. Emerg Infect Dis. 2015;21(8):1302-1310.

22. Morfin-Otero R, Rodríguez Noriega E, Dowzicky MJ. Antimicrobial susceptibility trends among gram positive and negative clinical isolates collected between 2005 and 2012 in Mexico: results from the tigecycline evaluation and surveillance trial. Ann Clin Microbial Antimicrob. $2015 ; 14: 53$

23. Jones RN, Guzmán Blanco M, Gales AC, et al. Susceptibility rates in Latin American Nations: report from a regional resistance surveillance program (2011). Braz J Infect Dis. 2013;17(6):672-681.

24. KPC Bacteria Kills 50 in Panama. Health Map: The Disease Daily; 2011.

25. Ministerio de salud República de Panamá. Resolución No. 0872 del 2 de septiembre de 2011 .

26. Girmenia C, Serrao A, Canichella M. Epidemiology of carbapenem resistant Klebsiella pneumoniae infections in Mediterranean countries. Mediterr J Hematol Infect Dis. 2016;8(1):e2016032.

27. Tang HJ, Hsieh CF, Chang PC, et al. Clinical Significance of Communityand Healthcare-Acquired Carbapenem Resistant Enterobacteriaceae Isolates. PLoS ONE. 2016;11(3):e0151897.

28. Dall Cortivo G, Gutverlet A, Agustini Ferreira J, et al. Antimicrobial resistance profiles and oxacillinase genes in carbapenem resistant Acinetobacter baumannii isolated from hospitalized patients in Santa Catarina, Brazil. Revista de Sociedade Brasileira de Medicina Tropical. 2015;48(6):699-705

29. El-Ageery SM, Abo-Shadi MA, Alghaithy AA, et al. Epidemiological investigation of nosocomial infection with multidrug-resistant Acinetobacter baumannii. Eur Rev Med Pharmacol Sci. 2012;16(13):1834-1839.

30. Podolsky SH, Powers JH. Regulating antibiotics in an era of resistance: the historical basis and continued need for adequate and well-controlled investigations. Ann Intern Med. 2015;163(5):386-388.

31. Avorn J, Solomon DH. Cultural and economic factors that (Mis) Shape antibiotic use: the no pharmacological basis of therapeutics. Ann Intern Med. 2000;133(2):128-135.

32. Gorgas Memorial Institute of Health Studies. Information system on drug spending in Panama. 2007-2014 [Internet]. Panama: Department of Health Technology Research and Evaluation; 2015.

33. Joseph A, Mahida N. Antimicrobial stewardship in secondary care: what are we trying to achieve?. Journal of Hospital Infection. 2016; 93(4):392-394.

34. Dhar S, Cook E, Oden M, et al. Building a successful infection prevention program. Key components, processes and Economics. Infect Dis Clin Am. 2016;30(3):567-589.

35. Castro Sanchez E, Holmes AH. Impact of organizations on healthcare associated infections. J Hosp Infect. 2015;89(4):346-350. 